\title{
Analysis and optimization of sensing agility in cognitive radio Bingcai Sun ${ }^{1}$, Shengliang Peng ${ }^{1 \mathrm{a}}$, Renyang $\mathrm{Gao}^{1}, \mathrm{Xi} \mathrm{Yang}^{2}$ \\ ${ }^{1}$ School of Information Science and Engineering, Huaqiao University, Xiamen 361021, China \\ ${ }^{2}$ School of Information Science and Engineering, Jishou University, Jishou 416000, China \\ aemail: peng.shengliang@gmail.com
}

Keywords: Sensing Agility; Cognitive Radio; Optimization

\begin{abstract}
Cognitive radio (CR) technology provides a new approach to solve the problem of wireless spectrum resource scarcity by allowing secondary users (SUs) to access the licensed spectrum in case that SUs do not interfere primary user (PU). One of the most challenging tasks in CR is spectrum sensing. This paper concentrates on the state change of PU's disappearance and analyzes the agility of spectrum sensing via evaluating its sensing time. Some expressions for sensing time are deduced. Based on these expressions, this paper also investigates how to optimize the sensing agility by choosing an appropriate detection period. Simulation results are provided to illustrate the validity of agility analysis and agility optimization in this paper.
\end{abstract}

\section{Introduction}

Despite being one of the most critical resources in wireless communications, the majority of available spectrum bands is allocated in a fixed way and not fully utilized according to recent reports [1] [2] [3] [4]. Cognitive radio is an effective approach to improve spectrum efficiency by enabling SU to reuse the spectrum band of PU [5]. In order to avoid interfering with PU, SU should sense whether the band is being used by PU [6].

Sensing accuracy, usually measured in terms of false alarm probability and missed detection probability, is a crucial index when evaluating the performance of spectrum sensing. Besides, the sensing agility is also of vital importance [7]. This index reflects the ability of SU to timely perceive state changes of PU on spectrum band, and ensures SU to take agile actions of either vacating or occupying the band once PU emerges or disappears, respectively. In [8], sensing agility is modelled by the number of detections required by SU to correctly determine PU's presence after PU emerges. If SU consumes small number of detections to perceive the emergence of PU, it could rapidly stop reusing the band and consequently do little interference to PU, resulting in high sensing agility. On the contrary, large number of detection leads to low sensing agility. In [9], the authors show that agility can be increased by allowing the SUs operating in the same band to cooperate and exploiting the inherent asymmetry in secondary networks.

This paper digs into the agility of spectrum sensing in cognitive radio. Different from previous research, it concentrates on PU's disappearance instead of PU's emergence. Sensing agility is thereby modelled by the sensing time required by SU to eventually find out PU's absence after PU disappears. This type of sensing agility has a significant impact as well. If SU could perceive the disappearance of PU within shorter time, more reuse opportunity can be grasped and higher spectrum efficiency can be achieved. Moreover, note that merely reducing the number of detections does not certainly do good to sensing agility. That is because overall sensing time depends not only on the number of detections but also on the period of each detection, and smaller detection number may be resulted from larger detection period. In this paper, detection period are taken into consideration and sensing agility is optimized by choosing an appropriate detection period. 


\section{Energy detection}

Assumed that energy detection method is used in each detection for spectrum sensing. SU determines whether PU exists or not with the observed energy $V$ of its received signal $y(k)$,

$$
V=\sum_{k=1}^{N}|y(k)|^{2}
$$

where $N$ denotes the number of samples used in energy detection.

Comparing $V$ with the decision threshold $\lambda$, whether the spectrum is occupied by PU (hypothesis $H_{1}$ ) or not (hypothesis $H_{0}$ ) can be expressed as

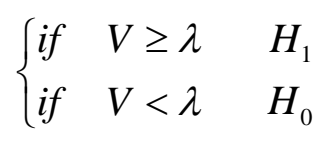

Without loss of generality, the channel noise is assumed to be additive white Gaussian noise (AWGN) with zero mean and unit variance, and PU signal is binary phase shift keying (BPSK) modulated. Then the false alarm probability $P_{f}$ and the missed detection probability $P_{m}$ are given by [10]

$$
\begin{aligned}
& P_{f}=Q\left(\frac{\lambda-N}{\sqrt{2 N}}\right) \\
& P_{m}=1-P_{d}=1-Q\left(\frac{\lambda-N(1+\gamma)}{\sqrt{2 N(1+2 \gamma)}}\right)
\end{aligned}
$$

where $\gamma$ is the received signal to noise ratio (SNR), $Q(x)=\int_{x}^{\infty} \frac{1}{\sqrt{2 \pi}} e^{-\frac{t^{2}}{2}} d t$ is the $Q$-function.

Generally, the time required by calculation and decision process can be neglected because it is much shorter than that of sampling process, so the time of each detection period approximately equals to its sampling time. If the sampling frequency is $f_{s}$, the detection period $T_{d}$ will be

$$
T_{d}=N / f_{s}
$$

Given sampling frequency $f_{s}$, detection period $T_{d}$ depends on sampling number $N$. Therefore, this paper mainly focuses on the sampling number to analyze sensing agility.

\section{Sensing agility analysis}

As described above, sensing agility reflects the ability of SU to sense the state changes of PU on license band, and can be measured by sensing time. This paper concentrates on the stage change that PU disappears and licensed band is no longer occupied by PU. Therefore, the sensing time here ranges from the moment when PU quits the licensed band to the moment when the idle band is perceived by SU.

Once PU's state changes from presence to absence, SU may not immediately get this information via energy detection as false alarm errors inevitably exist. Fortunately, the detection is periodically performed, and SU will eventually perceive the change after several times of detections. Assume the number of detections that SU consumes to perceive PU's disappearance is $L$, the sensing time can be expressed as

$$
T_{S}=L \cdot T_{d}=L \cdot N / f_{s}
$$

Note that false alarm error randomly happens, so how many detections are required is uncertain, that is, $L$ is a random variable. Consequently, major attention should be paid on its statistical mean. 
Considering the case $L=i$, in which SU determines the presence of PU in previous $i-1$ detections and finds out PU's absence in the ith detection. The probability of this case is

$$
P\{L=i\}=P_{f}^{i-1} \cdot\left(1-P_{f}\right)
$$

Then the statistical mean of $L$ can be deduced as

$$
E\{L\}=\sum_{i=1}^{\infty} i \cdot P\{L=i\}=\sum_{i=1}^{\infty} i \cdot P_{f}^{i-1} \cdot\left(1-P_{f}\right)=\frac{1}{1-P_{f}}
$$

So the average sensing time is

$$
E\left\{T_{s}\right\}=E\{L\} \cdot N / f_{s}=\frac{N / f_{s}}{1-P_{f}}
$$

Substituting (3) into (9), the expression can be rewritten as

$$
E\left\{T_{s}\right\}=\frac{N / f_{s}}{1-Q\left(\frac{\lambda-N}{\sqrt{2 N}}\right)}
$$

\section{Sensing agility optimization}

As shown in(6), the sensing agility is the product of detection period $T_{d}$ and detection number $L$. From the former point of view, smaller sample number $N$ is required according to (6), while from the latter point of view, larger sample number $N$ is needed according to (3) and (4). So there may exists an optimal sample number $N_{\text {opt }}$, which could make the average sensing time achieve minimum value and maximize the sensing agility.

Without loss of generality, considering the constant missed detection probability criterion that $P_{m}$ in each detection is confined to be a constant $\alpha$, the optimization problem can be expressed as follows

$$
\operatorname{Min}\left\{\frac{N / f_{s}}{1-Q\left(\frac{\lambda-N}{\sqrt{2 N}}\right)}\right\}
$$

Subject to : $P_{m}=1-Q\left(\frac{\lambda-N(1+\gamma)}{\sqrt{2 N(1+2 \gamma)}}\right)=\alpha$

Reforming of the optimization constraint(12), decision threshold $\lambda$ is given by

$$
\lambda=N(1+\gamma)+\sqrt{2 N(1+2 \gamma)} Q^{-1}(1-\alpha)
$$

Substituting (13) into the (11), the optimization problem with regard to $N$ is shown below

$$
\operatorname{Min}\left\{\frac{N / f_{s}}{1-Q\left(\frac{\gamma N+\sqrt{2 N(1+2 \gamma)} Q^{-1}(1-\alpha)}{\sqrt{2 N}}\right)}\right\}
$$

Given a certain sample number $N$, the average sensing time $E\left\{T_{S}\right\}$ can be calculated according to (14). Conducting numerical searching within the range of $N$, an optimal sample number $N_{\text {opt }}$ that minimizes $E\left\{T_{S}\right\}$ can be obtained. That is to say, in practical spectrum sensing scenarios, if using $N_{\text {opt }}$ samples in each detection, the average sensing time will be shortest, and 
the sensing agility is optimized correspondingly.

\section{Simulation and analysis}

This section provides Monte Carlo simulations and theoretical values to verify the correctness of conclusions deduced above. Simulation parameters are set as follows: $f_{s}=20$ Msamples $/ \mathrm{sec}$, $\alpha=0.5 \%$.

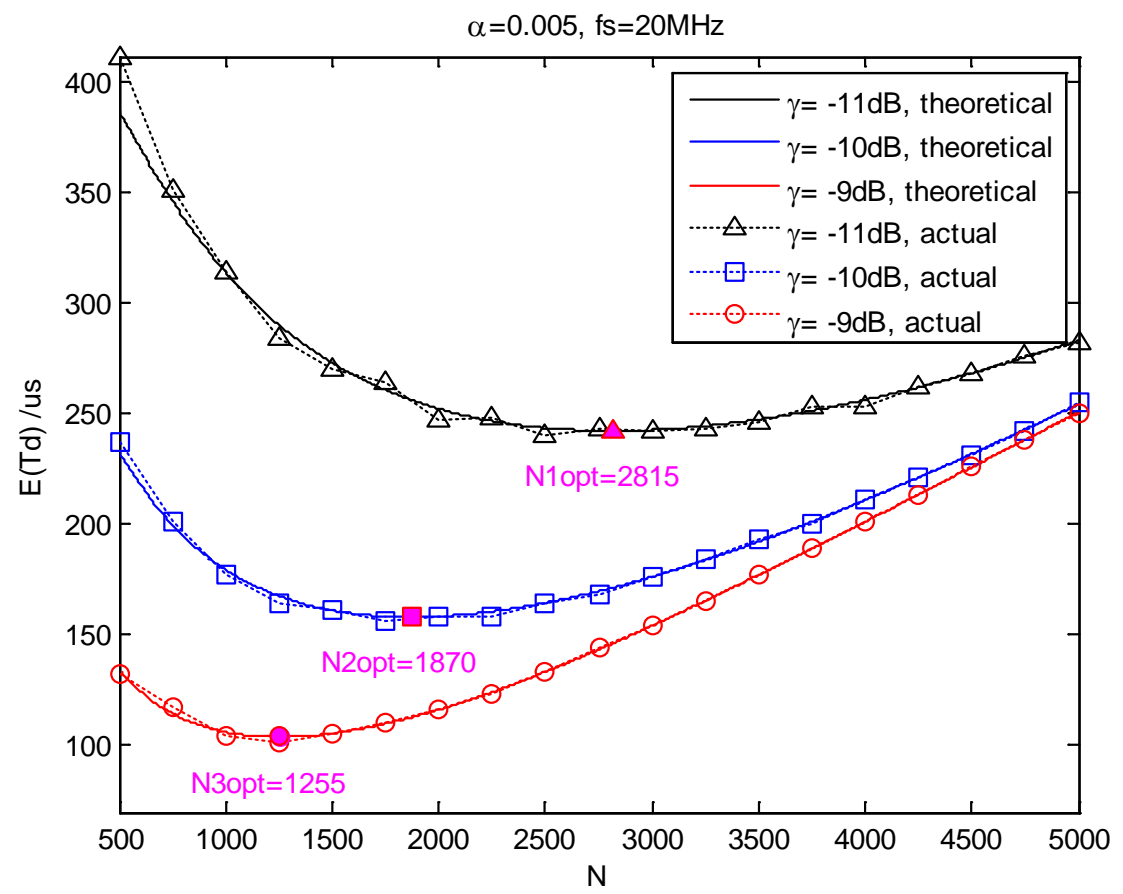

Fig. 1. Sensing agility versus sample number under different SNRs

Fig. 1 depicts the average sensing time $E\left\{T_{d}\right\}$ versus sample number $N$ under different SNRs ( $\gamma=-11 、-10 、-9 \mathrm{~dB})$, where "theoretical" curves are calculated by the theoretical results of this paper and "actual" curves are obtained from Monte Carlo simulations. It can be seen from Fig. 1, for each SNR, the actual curve is basically consistent with its corresponding theoretical curve. This phenomenon proves the correctness of our derivations. Furthermore, the trends of curves under different SNRs are similar with increasing $N$. They firstly decline, then reach the lowest point, and finally firstly rise, which demonstrates the existence of $N_{\text {opt }}$. Note that the deduced optimal sample number according to (15) is also plotted in this figure with the filled markers. Since these markers fall in accordance with the lowest points of corresponding Monte Carlo curves, the effectiveness of sensing agility optimization is examined.

\section{Conclusions}

This paper digs into the state change of PU's disappearance, and analyzes a new type of sensing agility. Evaluated in terms of average sensing time, the expression for sensing agility is deduced. Under the criterion of constant missed detection probability, the issue of optimizing sensing agility is also discussed, and a numerical method is provided to obtain the optimal sample number. If this optimal number is adopted in each detection, the agility of spectrum sensing can be minimized, resulting in higher spectrum efficiency.

\section{Acknowledgement}

This research is supported by the National Natural Science Foundation of China (No. 61201264, 61302095, 61362018) and Huaqiao University (No.12BS219, 13BS101). 


\section{References}

[1] FCC Spectrum Policy Task Force, Technical report [R]. Nov. 2002. 02-135.

[2] Yang J. Spatial channel characterization for cognitive radios [D]. University of California at Berkeley, USA, 2004.

[3] Cabric D, Mishra S M, Brodersen R W. Implementation issues in spectrum sensing for cognitive radios. Proceedings of the 2004 IEEE Conference record of the thirty-eighth Asilomar on signals, systems and computers, 2004 (1) 772-776.

[4] Shared Spectrum Company. Spectrum occupancy measurements [R]. Nov. 2005.

[5] Lu L, Zhou X, Onunkwo U, et al. Ten years of research in spectrum sensing and sharing in cognitive radio [J]. Eurasip Journal on Wireless Communications and Networking, 2012 (2012) $1-16$.

[6] Ganesan G, Li Y. Agility improvement through cooperative diversity in cognitive radio. Proceedings of the 2005 IEEE Global Telecommunications Conference [C]. St. Louis: Global Telecommunications Conference, 2005.2505-2509.

[7] Ganesan G, Li Y. Cooperative spectrum sensing in cognitive radio, part I: Two user networks [J]. IEEE Transactions on Wireless Communications, 2007 6(6) 2204-2213.

[8] Ganesan G, Li Y. Cooperative spectrum sensing in cognitive radio, part II: Multiuser networks [J]. IEEE Transactions on Wireless Communications, 2007 6(6) 2214-2222.

[9] Ganesan G, Li Y, Bing B, et al. Spatiotemporal sensing in cognitive radio networks[J]. IEEE Journal on Selected Areas in Communications, 2008 26(1) 5-12.

[10] S.-L. Peng, X. Yang, S. Shu, and X, Cao. Exploitation of temporal persistence for accuracy improvement in primary user detection[J]. IET Commun, 2009(4)1855-1864. 